\title{
Chemical reduction of hematite by sodium borohydride
}

\author{
V. G. de Resende • G. M. da Costa • \\ E. De Grave • L. Datas
}

Published online: 26 October 2006

(C) Springer Science + Business Media B.V. 2006

\begin{abstract}
Well-crystallized hematite was suspended in water and treated at roomtemperature (RT) with sodium borohydride. The product of the reaction is a highly magnetic black powder, which is stable at RT. The $\mathrm{NaBH}_{4}$ treatment converts about half of the hematite to an amorphous $\mathrm{Fe}-\mathrm{B}$ alloy and to a small fraction of sub-micron sized, amorphous metallic-Fe nodules. Heating at $400^{\circ} \mathrm{C}$ of this composite has resulted in the crystallization and/or oxidation of more than half of the amorphous $\mathrm{Fe}-\mathrm{B}$ phase to $\alpha$-Fe and $\mathrm{Fe}_{3} \mathrm{O}_{4}$ and $\mathrm{B}_{2} \mathrm{O}_{3}$, respectively. After treatment at $800^{\circ} \mathrm{C}$, the metallic $\mathrm{Fe}$ and the amorphous $\mathrm{Fe}-\mathrm{B}$ have completely vanished, and the resulting product consists of hematite and $\mathrm{FeBO}_{3}$ embedded in the matrix of $\alpha-\mathrm{Fe}_{2} \mathrm{O}_{3}$.
\end{abstract}

Key words Fe-B alloy $\cdot$ hematite $\cdot$ sodium borohydride $\cdot$ Mössbauer $\cdot$ spectroscopy

\section{Introduction}

The use of sodium borohydride for the production of amorphous transition-metal boron alloys (TM-B) has been the subject of intense research in recent years [1-3]. The soft route using $\mathrm{NaBH}_{4}$ offers several advantages with respect to other, more traditional methods (vapour deposition, melt spinning, sputtering, etc.) such as ease and promptness of the synthesis procedures and the possibility of preparing TM-B alloys with a broad variety in composition [4]. As far as it is known, all preparations methods reported so far in literature involve the addition of $\mathrm{NaBH}_{4}$ to a solution containing the metallic ions. The effect of the

V. G. de Resende • G. M. da Costa

Dep. de Química, Universidade Federal de Ouro Preto, 35400 Ouro Preto (MG), Brazil

V. G. de Resende • E. D. Grave ( $\square)$

Department of Subatomic and Radiation Physics, University of Ghent, 9000 Gent, Belgium e-mail: Eddy.DeGrave@UGent.be

L. Datas

CIRIMAT (LCMIE), Université Paul Sabatier, 31062 Toulouse, France 


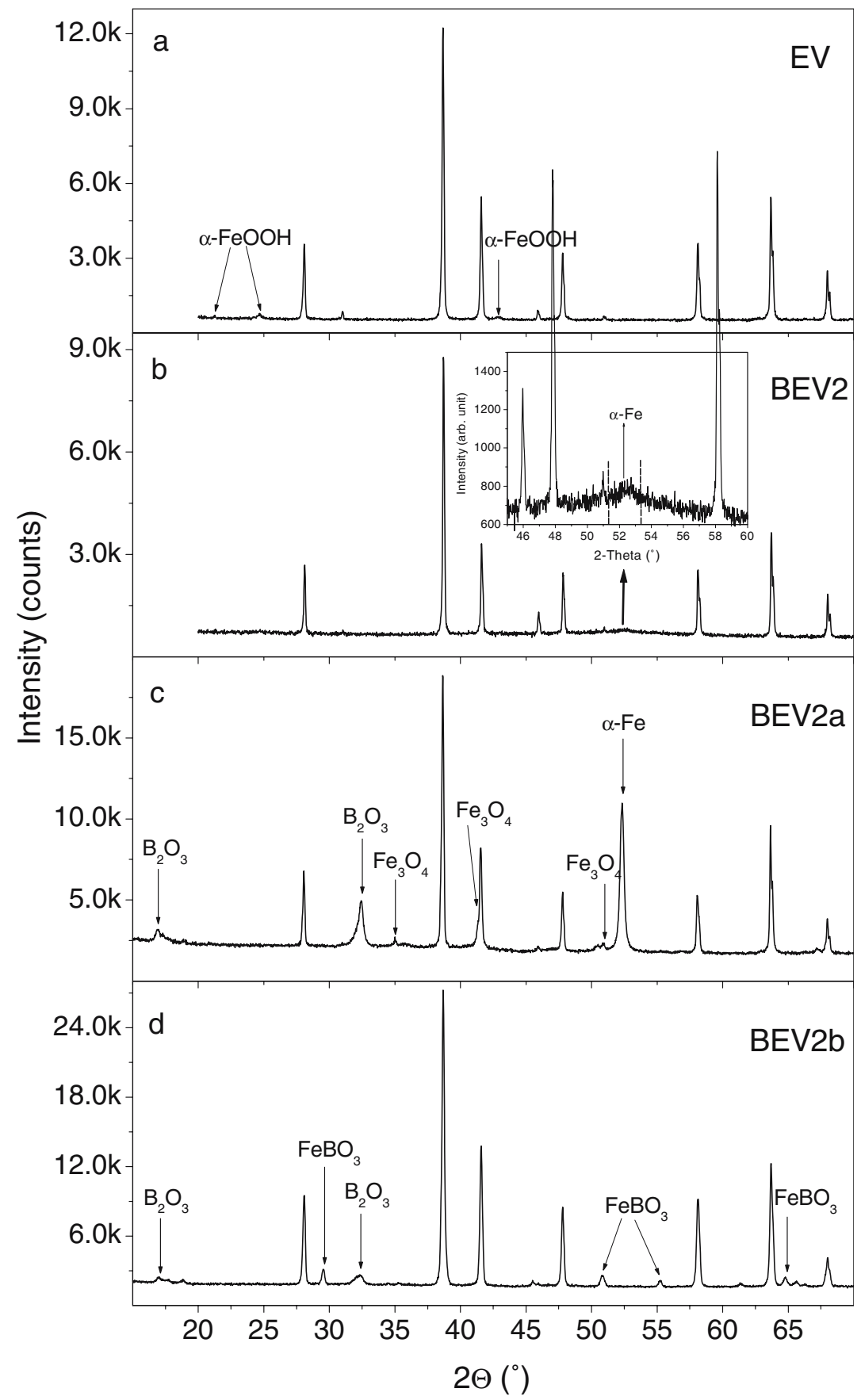

Figure 1 X-ray diffraction patterns of the original sample (EV), and after treatment with borohydride (BEV2). Samples BEV2a and BEV2b were obtained by heating BEV2 at 400 and at $800^{\circ} \mathrm{C}$, respectively. 
$\mathrm{pH}$, reaction time, and even of the sequence of the addition of the reactants on the composition and on the physicochemical properties of the obtained products have been examined by a number of authors [4-6]. On the other hand, the reaction of $\mathrm{NaBH}_{4}$ with solid particles has not been investigated, and the main objective of this work is to report for the first time the formation of amorphous $\mathrm{Fe}-\mathrm{B}$ alloy in aqueous medium by the reduction of hematite particles.

\section{Experimental}

The parent sample (hereafter called EV) involved in this study is an iron ore concentrate containing mainly hematite, with a negligible fraction of goethite. About $1.2 \mathrm{~g}$ of the sample was dispersed in $200 \mathrm{ml}$ of distilled water in which $4.0 \mathrm{~g}$ of $\mathrm{NaBH}_{4}$ had been dissolved prior to the dispersion. Hydrochloric acid $(100 \mathrm{ml}, 0.20 \mathrm{M})$ was added dropwise under vigorous stirring. Four portions of $0.8 \mathrm{~g}$ each of $\mathrm{NaBH}_{4}$ were added at regular intervals together with $100 \mathrm{ml}$ of $\mathrm{HCl}$. After $30 \mathrm{~min}$ of reaction, the solid was washed several times with distilled water and finally dried at $100^{\circ} \mathrm{C}$ (sample BEV2). Fractions of this sample were heated at $400^{\circ} \mathrm{C}$ (sample BEV2a) and $800^{\circ} \mathrm{C}(\mathrm{BEV} 2 \mathrm{~b}$ ) in static air during $1 \mathrm{~h}$.

$\mathrm{X}$-ray diffractograms $(\mathrm{XRD})$ were recorded in the range $15-70^{\circ}(2 \theta)$ with goniometer speed of $2^{\circ} / \mathrm{min}$ using a XRD-6000 Shimadzu diffractrometer equipped with a Co tube and Fe filter. Counts were registered every $0.02^{\circ}$.

Mössbauer spectra were collected with spectrometers operating at constant acceleration mode with triangular reference signals. ${ }^{57} \mathrm{Co}(\mathrm{Rh})$ sources were used. At room-temperature, both conventional transmission Mössbauer spectroscopy (TMS spectra) and integral lowenergy electron Mössbauer spectroscopy (ILEEMS spectra) have been applied. The latter variant probes the very top monolayers of the crystallites and gives information about those Fe species lying at distances of a few nanometers below the physical surfaces of the particles [7]. For samples BEV2 and BEV2a, TMS spectra were also recorded with the absorbers at a number of different temperatures between $12 \mathrm{~K}$ and RT. All Mössbauer spectra were computer-analysed in terms of model-independent distributions of hyperfineparameter values and numerical data quoted hereafter refer to maximum probability values. Isomer shifts are referenced with respect to $\alpha$-Fe at RT.

\section{Results and interpretation}

Sample EV has the typical macroscopic appearance of an iron ore with specularite being the dominant Fe phase. Specularite is a variety of hematite characterized by aggregates of silvery, specular hematite flakes or tabular crystals and as such shows a metallic brilliance. Hematite is weakly ferromagnetic at RT and for this reason, it is only slightly attracted by a hand magnet. On the other hand, sample BEV2 is black and highly magnetic. At first glance, the XRD pattern of BEV2 only shows the peaks corresponding to well-crystallised hematite (Figure 1b), but a closer examination of the diffractogram (inset Figure 1b) reveals the appearance of a shallow bump near $52^{\circ}(2 \theta)$, a position that corresponds to the principal diffraction line of $\alpha$-Fe. In addition, it can be noticed that the peaks corresponding to goethite (arrows in Figure 1a) are not present in BEV2, indicating that this phase has completely reacted with the borohydride. 


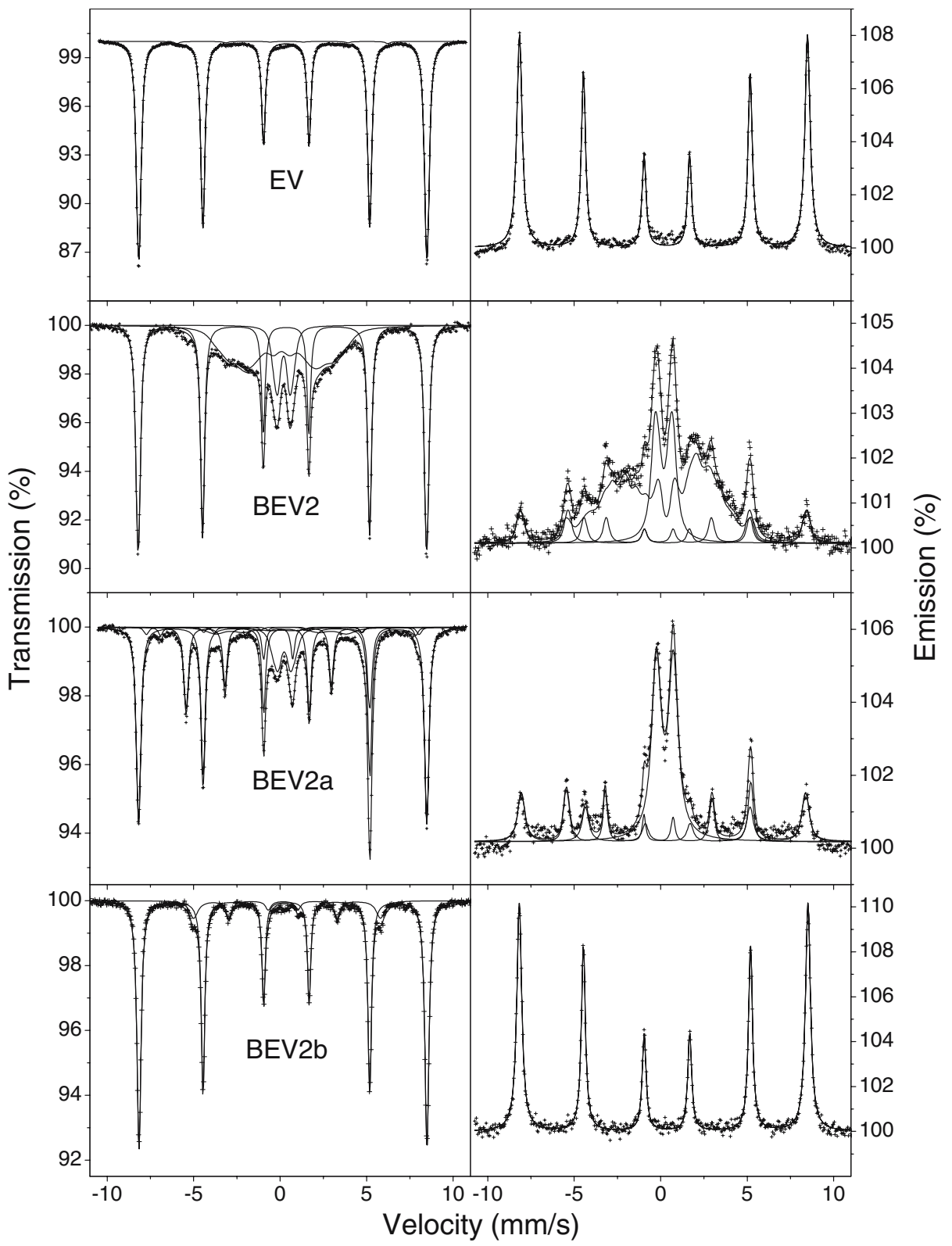

Figure 2 Room temperature transmission (left) and ILEEMS (right) Mössbauer spectra of the studied samples.

TMS and ILEEMS of sample EV at RT (Figure 2) yield one sharp sextet of which the relevant Mössbauer parameters, i.e., hyperfine field $H_{\mathrm{hf}}$ and quadrupole shift $2 \varepsilon_{\mathrm{Q}}$ (see Table I) are diagnostic of hematite. A very weak second sextet (area $S=1 \%$ relative to total spectral area) could be resolved from the TMS spectrum. Its hyperfine field and quadrupole shift at RT are $378 \mathrm{kOe}$ and $-0.26 \mathrm{~mm} / \mathrm{s}$, respectively, and can therefore be assigned to well-crystallized goethite, $\alpha-\mathrm{FeOOH}[8]$. 
Table I Mössbauer results of the samples measured at room temperature

\begin{tabular}{|c|c|c|c|c|c|c|c|c|c|c|c|c|c|c|c|c|}
\hline & \multicolumn{4}{|l|}{ EV } & \multicolumn{4}{|c|}{ BEV2 } & \multicolumn{4}{|c|}{ BEV2a } & \multicolumn{4}{|c|}{$\mathrm{BEV} 2 \mathrm{~b}$} \\
\hline & $H_{\mathrm{hf}}$ & $2 \varepsilon_{\mathrm{Q}}$ & $\delta$ & $S$ & $H_{\mathrm{hf}}$ & $2 \varepsilon_{\mathrm{Q}}^{\mathrm{b}}$ & $\delta$ & $S$ & $H_{\mathrm{hf}}$ & $2 \varepsilon_{Q}^{b}$ & $\delta$ & $S$ & $H_{\mathrm{hf}}$ & $2 \varepsilon_{\mathrm{Q}}$ & $\delta$ & $S$ \\
\hline \multicolumn{17}{|l|}{ Sextet I } \\
\hline Trans. & 517 & -0.20 & 0.37 & 100 & 517 & -0.21 & 0.35 & 54 & 517 & -0.22 & 0.37 & 56 & 516 & -0.20 & 0.37 & 87 \\
\hline ILEEMS & 516 & -0.19 & 0.37 & 100 & 515 & $-0.20^{\mathrm{a}}$ & $0.37^{\mathrm{a}}$ & 11 & 511 & -0.25 & 0.39 & 23 & 518 & -0.19 & 0.37 & 100 \\
\hline \multicolumn{17}{|l|}{ Sextet II } \\
\hline Trans. & - & - & - & - & 185 & $0.00^{\mathrm{a}}$ & 0.19 & 35 & 237 & $0.00^{\mathrm{a}}$ & 0.16 & 7 & - & - & - & - \\
\hline ILEEMS & - & - & - & - & 208 & $0.00^{\mathrm{a}}$ & 0.24 & 53 & - & - & - & - & - & - & - & - \\
\hline \multicolumn{17}{|l|}{ Sextet III } \\
\hline Trans. & - & - & - & - & - & - & - & - & 329 & -0.01 & -0.01 & 22 & - & - & - & - \\
\hline ILEEMS & - & - & - & - & 327 & $0.00^{\mathrm{a}}$ & $0.00^{\mathrm{a}}$ & 12 & 331 & 0.00 & 0.00 & 20 & - & - & - & - \\
\hline \multicolumn{17}{|c|}{ Sextet IV/V } \\
\hline Trans. & - & - & - & - & - & - & - & - & 487 & $0.00^{\mathrm{a}}$ & $0.26^{\mathrm{a}}$ & 2 & - & - & - & - \\
\hline ILEEMS & - & - & - & - & - & - & - & - & 457 & $0.00^{\mathrm{a}}$ & 0.58 & 2 & - & - & - & - \\
\hline \multicolumn{17}{|l|}{ Sextet VI } \\
\hline Trans. & - & - & - & - & - & - & - & - & - & - & - & - & 335 & 0.24 & 0.38 & 13 \\
\hline ILEEMS & - & - & - & - & - & - & - & - & - & - & - & - & - & - & - & - \\
\hline \multicolumn{17}{|l|}{ Doublet } \\
\hline Trans. & - & - & - & - & - & 0.63 & 0.31 & 11 & - & 0.64 & 0.35 & 11 & - & - & - & - \\
\hline ILEEMS & - & - & - & - & - & 0.93 & 0.36 & 24 & - & 1.00 & 0.35 & 57 & - & - & - & - \\
\hline
\end{tabular}

For the doublet, $\Delta E_{\mathrm{Q}}$ means the quadrupole splitting $(\mathrm{mm} / \mathrm{s})$. The values of isomer shifts are with reference to metallic iron.

$H_{\mathrm{hf}}$ maximum-probability hyperfine fields $(\mathrm{kOe}), 2 \varepsilon_{\mathrm{Q}}$ quadrupole shifts $(\mathrm{mm} / \mathrm{s}), \delta$ isomer shifts $(\mathrm{mm} / \mathrm{s})$ and $S$ relative areas $(\%)$ of the various spectral components resolved from the room-temperature transmission and ILEEMS Mössbauer spectra

${ }^{\text {a }}$ Fixed parameter

${ }^{\mathrm{b}} \Delta E_{\mathrm{Q}}$ for the doublet component

The RT TMS of sample BEV2 presents, in addition to the sharp hematite sextet (relative spectral area $S=54 \%$ ), a very broad sextet (35\%, henceforward termed sextet II) and a doublet (11\%). In fitting this spectrum, the area ratio of outer lines to middle lines to inner lines for the broad sextet was forced to equal 3:2:1. From the results of the effect of temperature on the TMS of BEV2 (not shown) and the numerical results derived from these spectra (also not shown), it is obvious that sextet II remains considerably broadened, even at $12 \mathrm{~K}$, and that its contribution slightly increases with lowering of temperature. As for the doublet, it seems to persist down to temperatures as low as $12 \mathrm{~K}$, but it is quite possible that it would completely vanish on further lowering the temperature towards $0 \mathrm{~K}$. Due to the weak contribution of this doublet, its Mössbauer parameters are ill defined and the adjusted hyperfine-parameter values inevitably are uncertain, thus explaining the unreal fluctuations in the obtained temperature variations of these values. It is believed, though, that the consistent decrease of the doublet's relative area with decreasing temperature, the amount by which fully accounting for the increase of the area of sextet II, is not unreal.

The above mentioned findings suggest that sextet II and doublet, as resolved from the TMS spectra of BEV2, both are due to one and the same structural phase of which the magnetic order-disorder-transition temperature is spread out over a very broad temperature range. Such behaviour one may expect to be observed for magnetic small-particle or amorphous systems with a broad distribution in particle size or composition, respectively. Based upon published data referring to amorphous Fe-B alloys [4], upon the type of 
elements present in the starting mixture and upon the observation that no diffraction lines other than those from $\alpha-\mathrm{Fe}_{2} \mathrm{O}_{3}$ and $\alpha$-Fe are obvious, one may assign sextet II and doublet to an amorphous $\mathrm{Fe}-\mathrm{B}$ alloy, henceforward referred to as the a-( $\mathrm{Fe}, \mathrm{B})$ phase. The global composition of this phase is $\mathrm{Fe}_{0.9} \mathrm{~B}_{0.1}$, as determined by wet chemical analysis and ICP.

Finally, the $2 \varepsilon_{\mathrm{Q}}$ data for the hematite sextet indicate that the Morin transition of the hematite phase occurs at a temperature well above $220 \mathrm{~K}$, implying a high degree of crystallinity of the parent ferric oxide with minor or no substitutions of the ferric cations by other species such as $\mathrm{Al}, \mathrm{Mn}, \mathrm{Ti}$, etc. [9], which are common in natural hematites.

The ILEEMS spectrum of BEV2 at RT shows that the amount of hematite in the surface layers, as quantified by the relative spectral area $S=11 \%$ of the hematite component appearing in the ILEEMS, is much less than in the bulk, where $S=54 \%$. On the other hand, the area of doublet has nearly doubled from $11 \%$ (bulk) to $24 \%$ (surface). Two other components are clearly identified in this ILEEMS spectrum: the broad sextet (53\%) corresponding to sextet II in the TMS spectra and a third sextet, designated as sextet III in Table I, which obviously is due to metallic iron (12\%). The observation that both sextet II and doublet components in the ILEEMS spectrum are enhanced with respect to their respective counterparts in the TMS spectrum, is consistent with the suggestion that they are indeed attributable to the same constituent, which thus is most likely initially formed in the surface layers of the hematite grains.

The XRD patterns of the samples obtained by heating BEV2 at $400^{\circ} \mathrm{C}(\mathrm{BEV} 2 \mathrm{a}$, highly magnetic) and $800^{\circ} \mathrm{C}(\mathrm{BEV} 2 \mathrm{~b}$, non-magnetic) are reproduced in Figure 1c and d, respectively. The most noticeable aspect is the presence of a sharp peak corresponding to metallic iron in $\mathrm{BEV} 2 \mathrm{a}$, and the absence of this peak upon heating at $800^{\circ} \mathrm{C}$. Additional diffraction lines, due to magnetite, $\mathrm{Fe}_{3} \mathrm{O}_{4}$, and $\mathrm{B}_{2} \mathrm{O}_{3}$ in sample $\mathrm{BEV} 2 \mathrm{a}$ and to $\mathrm{FeBO}_{3}$ and $\mathrm{B}_{2} \mathrm{O}_{3}$ in $\mathrm{BEV} 2 \mathrm{~b}$, are readily recognized.

The TMS spectra of BEV2a (see Figure 2) clearly show the presence of $\alpha$-Fe and $\mathrm{Fe}_{3} \mathrm{O}_{4}$, (sextets III and IV/V, respectively, in Table I) along with hematite and the iron phases that give rise to the broad sextet II and a weak doublet. The fraction of the total Fe atoms that forms the hematite phase has remained unchanged from that in the parent sample BEV2 prior to heating. In contrast, the contribution of sextet II to the total spectrum of BEV2a, i.e., $\sim 10 \%$, is considerably lower as compared to BEV2. Its Mössbauer parameters are ill defined and consequently, as one can notice from the numerical data, they bear a large scatter for the various temperatures. Remarkably, the relative area of the doublet has remained unaffected by the heating at $400^{\circ} \mathrm{C}$, and as for BEV2 tends to zero when the temperature lowers towards $0 \mathrm{~K}$. Further, the amount of metallic Fe has doubled as a result of the heating.

The ILEEMS spectrum of BEV2a (Figure 2) shows a predominant contribution from the doublet. As for BEV2, the hematite fraction in the surface layers is less than that in the bulk, whilst no difference in that respect is observed for the metallic-Fe fraction. An additional component that would correspond to the sextet II in the TMS spectra and a magnetite contribution are not resolved. Both the transmission and ILEEMS spectra of BEV2b show the predominant sharp sextet for hematite, but in the transmission data the presence of a second sextet is clearly recognized. Its parameters are similar to those of $\mathrm{FeBO}_{3}[10]$, which are consistent with the XRD pattern of this sample. The borate phase does not appear in the ILEEMS spectrum, implying that the borate grains are embedded in the hematite matrix.

More detailed results concerning this work will be published in a forthcoming paper. 
Acknowledgements This work was partially funded by CNPq and Fapemig (Brazil), and by the Fund for Scientific Research - Flanders, Belgium.

One of us (V. G. de Resende) thanks the Program Alban, the European Union Programme of High Level Scholarships for Latin America, scholarship no. E04M034189BR.

\section{References}

1. Dragieva, L.D., Stoynov, Z.B., Klabunde, K.J.: Scr. Mater. 44, 2187 (2001)

2. Foster, G.L., Barquín, L.F., Cohen, N.S., Pankhurst, Q.A., Parkin, I.P.: J. Mater. Process. Technol. 92-93, 525 (1999)

3. Petit, S., David, K., Doumerc, J.P., Grenier, J.C., Seguelong, T., Pouchard, M.: C. R. Acad. Sci. Paris Série IIc 517, (1998)

4. Linderoth, S., Mørup, S.: J. Appl. Phys. 69, 5256 (1991)

5. Saida, J., Ghafari, M., Nakamura, Y., Inoue, A., Masumoto, T.: Nucl. Instrum. Methods Phys. Res. B76, 223 (1993)

6. Song, X., Yusheng, X., Huali, J., Qing, X.: Nucl. Instrum. Methods Phys. Res. B76, 260 (1993)

7. De Grave, E., Vandenberghe, R.E., Dauwe, C.: Hyperfine Interact. 161, 147 (2005)

8. De Grave, E., Vandenberghe, R.E.: Hyperfine Interact. 28, 643 (1986)

9. De Grave, E., Bowen, L.H., Vochten, R., Vandenberghe, R.E.: J. Magn. Magn. Mater. 72, 141 (1988)

10. Bayukov, O.A., Abd-Elmeguid, M.M., Ivanova, N.B., Kazak, N.V., Ovchinnikov, S.G., Rudenko, V.V.: Phys. Solid State 46(6), 1088 (2004) 